\title{
Sichere Hilfe gegen Schweißbildung
}

— Habituelles übermäßiges Schwitzen ist zwar gesundheitlich unbedenklich, bedeutet jedoch für die Betroffenen trotzdem eine große Belastung. Als kostengünstige Alternative zu invasiven Verfahren wie Entfernung von Schweißdrüsen oder Botulinuminjektionen bietet sich ein Antitranspirans mit Aluminiumchlorid an. In geeigneter Konzentration bewirkt diese Substanz eine Verengung der Schweißkanäle und reduziert so die Bildung von Schweiß.

Vielfach kommt es bei der Anwendung jedoch zu Hautreizungen und damit zum Abbruch der Behandlung. Mit Odaban ${ }^{\circledR}$ Spray steht ein Therapeutikum zur Verfügung, bei dem entzündliche Hautreaktionen vermieden werden können. Herkömmliche Produkte mit Aluminiumchlorid werden meist als Roll-ons oder in Tupfspendern angeboten, die das Eindringen von Wasser ermöglichen. Dabei kann Salzsäure entstehen, die eine Reizung der Haut bewirkt. Der neue Spray ist dagegen hermetisch versiegelt, sodass der Inhalt nicht mit Wasser in Berührung kommen kann. Zusätzlich enthält er Silikonöl, das die Haut vor dem Austrocknen schützt und auch eine gleichmäßige Benetzung des Wirkstoffs auf der Haut fördert.

Ein weiteres Produkt aus der Odaban ${ }^{\circledast}$-Reihe sind die gleichnamige Handlotion, die neben
Aluminiumchlorid auch pflegende Substanzen wie Avocadoöl, Myrthenöl, Tocopherol und hochwertige Wachse enthält. Produkte, die Aluminiumchlorid enthalten, sollten nachts angewendet werden, wenn der Patient nur wenig schwitzt. Das Aluminiumchlorid bindet sich an die Proteine der Schweißkanäle; überschüssige Reste können am nächsten Morgen abgewaschen werden. Wichtig ist, eine Überdosierung mit Aluminiumchlorid zu vermeiden. In diesem Fall könnte ein kompletter Verschluss der Schweißkanäle mit einem darauf folgenden „Dammbruch" und überschießender Schweißsekretion resultieren.

Nach Informationen von MDM Healthcare Deutschland

\section{Systemische Psoriasis-Therapie frühzeitig initiieren}

- Psoriasis liegt oft als Erkrankung mit bereits systemischen Veränderungen vor. Der inflammatorische Prozess geht mit erhöhten kardiovaskulären Risiken einher, betonte Prof. Dr. Kristian Reich, Hamburg: „Ein Mann von 30 Jahren mit einer schweren Psoriasis hat ein dreifach erhöhtes Risiko, einen Herzinfarkt zu erleiden". Das metabolische Syndrom tritt bei den Betroffenen fast dreimal, der Diabetes mellitus Typ 2 doppelt so häufig auf wie bei Patienten ohne Psoriasis.

Nach akutellen Leitlinien ist eine systemische Therapie ab einer mittelschweren Ausprägung der Erkrankung indiziert - wobei jedoch kein exakter Schwellenwert definiert ist. Reich verwies auf einen „Graubereich", der bei vielen Ärzten Verunsicherung auslöst. Häufig zeigt eine topische Therapie nicht den gewünschten Erfolg, der als eine mindestens 50\%ige Reduktion des Psoriasis Area and Severity Index (PASI50) definiert ist.

In solchen Fällen kann eine als noch leicht beurteilte Psoriasis bereits als systemisch behandlungsbedürftig eingestuft werden, so Reich. Vor kurzem wurde ein europäisches Konsensuspapier veröffentlicht, das weitere Kriterien enthält, ab wann eine systemische Therapie indiziert ist (Mrowietz U et al. Arch Dermatol Res 2011; 25:
1-13). Darunter zählen beispielsweise die Beteiligung großer Teile der Kopfhaut oder anderer sichtbarer Bereiche, des Genitalbereichs, schwere Nagelpsoriasis oder therapierefraktäre Plaques.

Mit Fumaderm ${ }^{\oplus}$, so Reich, steht ein systemisches Präparat zur Verfügung, das auch in der Langzeittherapie eine gute Wirksamkeit und Verträglichkeit aufweist. Das bereits für die mittelschwere Psoriasis zugelassene Präparat besitzt auch ein gutes Kosten-Nutzen-Profil. Reimund Freye

Pressekonferenz "Umdenken bei Psoriasis: frühzeitig systemisch behandeln. Neue Ergebnisse zu Lebensqualität, Compliance und Folgen der Psoriasis" anlässlich der 46. DDGTagung, Dresden, 30. März 2011 (Veranstalter: Biogen Idec)

\section{Neues Instrument gegen aktinische Keratosen}

- Für Patienten mit aktinischer Keratose wird in Kürze eine neue, nicht-invasive Therapieoption zur Verfügung stehen. Die lackbildende Lösung zum Auftragen aus niedrig dosiertem 5-Fluorouracil (0,5\%) sowie Salicylsäure (10\%) wird voraussichtlich ab Sommer 2011 unter dem Namen Actikerall ${ }^{\circledR}$ verfügbar sein und die bisherigen, ausschließlich invasiv-physikalischen Behandlungsansätze bei hyperkeratotischen Läsionen ergänzen. Prof. Dr. Eggert Stockfleth, Berlin, nannte als das wichtigste
Resultat der Zulassungsstudie: „Die neue Substanz hat eine histologische Clearance bei $72 \%$ der vordefinierten hyperkeratotischen Läsionen erzielt."

Mit dem Vergleichspräparat aus Diclofenac (3\%) plus Hyaluronsäure - dem Standardmedikament gegen aktinische Keratosen waren es 59\% der Läsionen. Die dimethylsulfoxidhaltige Salbengrundlage des neuen Produkts als „Placebo" erzielte eine histologische Clearance bei 44\% der Läsionen. In der Beurteilung der klinischen Besserung ergab sich für die drei Grupen ein vergleichbares abgestuftes Bild. In die Zulassungsstudie waren 470 Patienten eingeschlossen, die bis zu zwölf Wochen therapiert und anschließend über acht Wochen beobachtet wurden. Als unerwünschte Nebenwirkungen zeigten sich vor allem lokale Reizungen. Die Lösung kann zur Behandlung auch mit der bisherigen Standardtherapie kombiniert werden. Simone Reisdorf

Seminar "Aktinische Keratosen: Neues aus der Forschung" anlässlich der 46. DDG-Tagung, Dresden, 31. März 2011 (Veranstalter: Almirall Hermal) 